University of Nebraska - Lincoln

DigitalCommons@University of Nebraska - Lincoln

Faculty Publications: Department of Entomology

2000

\title{
Influence of Corn Phenology on Adult Western Corn Rootworm (Coleoptera: Chrysomelidae) Distribution
}

\author{
Stephanie J. Darnell \\ University of Nebraska-Lincoln \\ Lance J. Meinke \\ University of Nebraska-Lincoln, Imeinke1@unl.edu \\ Linda J. Young \\ University of Nebraska-Lincoln, lyoung3@unl.edu
}

Follow this and additional works at: https://digitalcommons.unl.edu/entomologyfacpub

Part of the Entomology Commons

Darnell, Stephanie J.; Meinke, Lance J.; and Young, Linda J., "Influence of Corn Phenology on Adult Western Corn Rootworm (Coleoptera: Chrysomelidae) Distribution" (2000). Faculty Publications: Department of Entomology. 264.

https://digitalcommons.unl.edu/entomologyfacpub/264

This Article is brought to you for free and open access by the Entomology, Department of at DigitalCommons@University of Nebraska - Lincoln. It has been accepted for inclusion in Faculty Publications: Department of Entomology by an authorized administrator of DigitalCommons@University of Nebraska - Lincoln. 


\title{
Influence of Corn Phenology on Adult Western Corn Rootworm (Coleoptera: Chrysomelidae) Distribution
}

\author{
STEPHANIE J. DARNELL, LANCE J. MEINKE, AND LINDA J. YOUNG ${ }^{1}$ \\ Department of Entomology, University of Nebraska, Lincoln, NE 68583-0816
}

\begin{abstract}
Environ. Entomol. 29(3): 587-595 (2000)
ABSTRACT Field studies were designed to more clearly determine how adult western corn rootworm, Diabrotica virgifera virgifera LeConte, population distribution patterns are altered over time by changes and contrasts in corn (Zea mays L.) plant phenology using whole-plant beetle counts as the sampling tool. In 1994, studies were conducted in a model system consisting of a late-planted corn strip placed in the middle of an early-planted cornfield. The system was replicated over three fields. Large-scale variation was modeled using trend-surface regression analysis to describe the relationship between beetle counts and distance from the center of the late-planted strip. In each field, the beetle distribution became greatly skewed toward the late-planted strip when the strip was either in the tassel or silk stage and the surrounding field was $\geq$ blister stage. In 1995, studies were conducted in and at the interface of two adjacent cornfields that were planted $9 \mathrm{~d}$ apart. Count data were analyzed to quantify population density changes at different sampling locations within and among fields over time as crop phenology changed. Rapid positive or negative changes in beetle densities occurred within and among fields as contrasts in corn phenology changed. In both years, the stability of the beetle distribution was strongly influenced by the length of time that a contrast in plant phenology was maintained between adjacent patches of corn. Directional movements of beetles toward pollinating corn and associated semiochemicals could be inferred from both 1994 and 1995 data analyses. Data suggest that contrasts in crop phenology at the interface and among cornfields should be considered when developing beetle sampling programs and interpreting scouting data to improve the accuracy of rootworm management decisions.
\end{abstract}

KEY WORDS movement, corn silks, sampling, Diabrotica virgifera virgifera, population dynamics

THE WESTERN CORN rootworm, Diabrotica virgifera virgifera LeConte, is a widely distributed pest of field corn (Zea mays L.) east of the Rocky Mountains in the United States (Chiang 1973). The larval stage can cause economic damage by feeding on corn roots which results in decreased plant growth and reduced yield (Chiang 1973, Apple et al. 1977, Levine and Oloumi-Sadeghi 1991, Spike and Tollefson 1991, Godfrey et al. 1993, Urias 1998). Yield losses and control expenses attributed to corn rootworms annually cost producers millions of dollars.

Insecticides are often applied when corn is planted in a field for two or more successive years (continuous corn) to manage corn rootworms. A common approach is to apply a soil insecticide at planting time or first cultivation to control larvae in the root zone (Mayo and Peters 1978). An alternative strategy is to use a beetle spray program to suppress adult corn rootworm populations and reduce egg laying so that larval populations the following year will not cause economic loss (Pruess et al. 1974, Meinke 1995). Although the larval stage is responsible for most of the economic damage in field corn, the adult stage is the focus of most sampling programs. Egg and larval sam-

\footnotetext{
${ }^{1}$ Department of Biometry, University of Nebraska, Lincoln, NE 68583.
}

pling techniques are rarely used to estimate rootworm populations and to make management decisions in commercial field corn because of the intensive amount of time and labor involved in collecting and processing soil samples (Bergman et al. 1981, Ruesink 1986, Tollefson 1990). Adult sampling techniques have been successfully used to estimate corn rootworm population density levels and to develop economic thresholds (Roselle 1977, Stamm et al. 1985, Tollefson 1990) for larval or adult control situations. Currently, the visual or whole-plant beetle count sampling method (Hein and Tollefson 1985) is most commonly used by agriculture professionals to make rootworm management decisions.

A good understanding of adult corn rootworm behavior is needed to effectively take beetle counts on plants and subsequently interpret sampling data. Important factors influencing population distributions of corn rootworm beetles are adult mobility and hostplant phenology. Western corn rootworm beetles are very mobile insects with both trivial movement (Naranjo 1991, 1994) and migration (Coats et al. 1986, Grant and Seevers 1989, Naranjo 1990) events impacting spatial dynamics over time. When movement occurs among cornfields (Hill and Mayo 1980), the majority of the dispersal activity appears to be by females 
(Hill and Mayo 1974, Godfrey and Turpin 1983, Naranjo 1991).

Western corn rootworm beetle movement is often highly correlated with the relative attractiveness of food sources. Preferred food sources have been identified as pollen, corn silks, and corn kernels (Ball 1957, Hintz and George 1966, Ludwig and Hill 1975). Volatiles in maize silks act as adult attractants (Prystupa et al. 1988), and some silk compounds are electroantennogram-active (Abou-Fakhr et al. 1996, Hibbard et al. 1997). Hill and Mayo (1974) examined the idea of using a late-planted trap crop for corn rootworm management. They observed that the trap crop attracted western corn rootworm beetles from the surrounding early-planted corn, and increased oviposition in the trap area, therefore reducing the larval injury potential in corn planted near the trap area the following year.

Increasing our understanding of the impact of corn phenology on beetle movement and associated distributional patterns can help to improve the accuracy of decision making when scouting or using adult management strategies. This article presents the results of field experiments that were designed to more clearly characterize the large-scale spatial distribution of adult western corn rootworms in response to temporal changes and contrasts in corn plant phenology. Whole-plant beetle counts were used as the sampling tool to generate data sets in both a model system and commercial cornfields.

\section{Materials and Methods}

1994 Field Experiment. A model system was developed that consisted of a 16-row strip $(0.76 \mathrm{~m}$ row spacing) of late-planted corn (late-planted strip) placed in the center of an earlier-planted cornfield so that differences in corn phenology occurred over time. The model system was replicated in three continuous cornfields ranging from 12.2 to 32.4 ha. Fields 1 and 3 were irrigated, and field 2 was dryland production. The cornfields were planted between 25 and 27 April 1994 (Hybrids: fields 1 and 3, Pioneer 3162; field 2, Hoegemeyer 2700), and the late-planted strips were planted between 24 and 25 May 1994 (Hybrids: fields 1 and 3, Pioneer 3162; field 2, Hoegemeyer 2700). The experiment was conducted at the University of Nebraska Agricultural Research and Development Center, near Mead, NE.

Beetle emergence patterns were determined by monitoring emergence cages that were centered over one plant (Hein et al. 1985), but covered roots the equivalent of two plants per cage. In each field, six cages were placed on each side of the late-planted strip (within $20 \mathrm{~m}$ of the strip) in transects across the earlier planted cornfield. Beetles were collected from the cages at 3- to 7-d intervals during the emergence period, 5 July to 26 August. Western corn rootworm adults were subsequently separated by sex in the laboratory.

In each field, beetles on entire plants were counted (whole-plant counts) as described by Hein and Tollef-

\section{A. 1994}

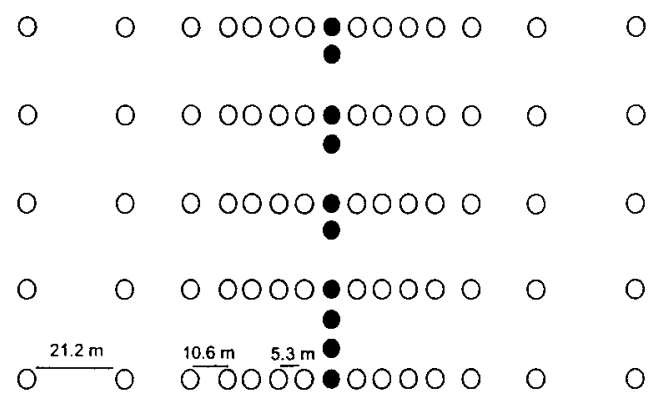

B. 1995

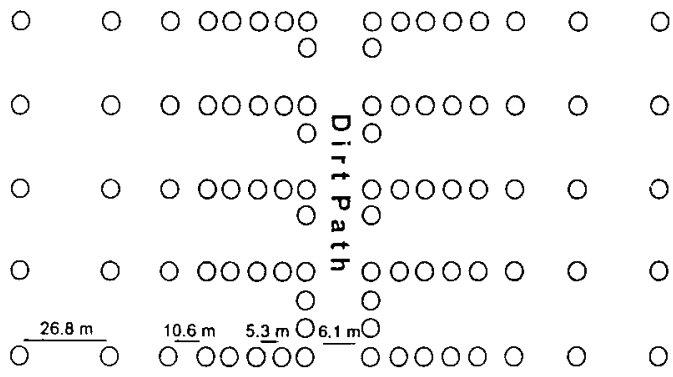

Fig. 1. Sampling grids used in 1994 (A) and in 1995 (B). Black circles represent sampling in late-planted strip in 1994.

son (1984) and Darnell et al. (1999) at 4- to 7-d intervals from 20 July through 7 September 1994 to estimate population densities. Counts were taken in the morning on each sampling date. A sampling grid containing 80 sample sites per field was used for whole-plant counts. The sites ranged from 5.3 to $74.2 \mathrm{~m}$ on either side of the midpoint of the late-planted strips with the greatest number of sites per unit area in and near the late-planted strips (Fig. 1A). In field 2, the north side of the strip had one less sampling distance than the south side because of field size constraints. Two randomly chosen plants $<1 \mathrm{~m}$ apart were examined per site for western corn rootworm beetles on each sampling date. Mean whole-plant counts per site were used for analyses.

1995 Field Experiment. Field experiments were conducted in two adjacent continuous cornfields that were divided by a dirt path $6.1 \mathrm{~m}$ wide. A 9-d difference in planting dates between fields (field 4, 16 ha planted 25 May 1995; field 5, 6 ha planted 3 June 1995) resulted in differences in plant phenology between the fields over time. The surrounding fields consisted of soybeans, Glycine max (L.) Merrill, and the closest cornfield to the experimental fields was $0.4 \mathrm{~km}$ away. The study site was on a farm near Valparaiso, NE.

Beetle emergence patterns were determined as previously described. Six cages were placed in transects across each field, and beetles were collected from the cages at 5- to 7-d intervals from 19 July to 28 August 
Table 1. 1994 western corn rootworm emergence profiles

\begin{tabular}{|c|c|c|c|c|c|c|c|c|c|}
\hline \multirow{3}{*}{ Date } & \multicolumn{3}{|c|}{ Field 1} & \multicolumn{3}{|c|}{ Field 2} & \multicolumn{3}{|c|}{ Field 3} \\
\hline & \multicolumn{2}{|c|}{$\begin{array}{l}\text { Cumulative } \\
\text { emergence }\end{array}$} & \multirow{2}{*}{$\begin{array}{l}\text { Cumulative } \\
\% \text { emergence }\end{array}$} & \multicolumn{2}{|c|}{$\begin{array}{l}\text { Cumulative } \\
\text { emergence }\end{array}$} & \multirow{2}{*}{$\begin{array}{l}\text { Cumulative } \\
\% \text { emergence }\end{array}$} & \multicolumn{2}{|c|}{$\begin{array}{l}\text { Cumulative } \\
\text { emergence }\end{array}$} & \multirow{2}{*}{$\begin{array}{l}\text { Cumulative } \\
\% \text { emergence }\end{array}$} \\
\hline & No. $\widehat{\sigma}$ & No. 우 & & No. $\sigma$ & No. 9 & & No. $\delta$ & No. 9 & \\
\hline 5 July & 2 & 0 & 2.4 & 19 & 1 & 13.5 & 1 & 0 & 0.8 \\
\hline 8 July & 13 & 0 & 15.9 & 34 & 7 & 27.7 & 18 & 1 & 14.7 \\
\hline 12 July & 21 & 4 & 30.5 & 56 & 28 & 56.8 & 28 & 11 & 30.2 \\
\hline 18 July $^{a}$ & 27 & 14 & 50.0 & 77 & 57 & 90.5 & 43 & 26 & 53.5 \\
\hline 26 July & 30 & 33 & $76.8^{b}$ & 77 & 60 & $92.6^{b}$ & 62 & 48 & 85.3 \\
\hline 2 Aug. & 33 & 42 & 91.5 & 81 & 61 & 95.9 & 66 & 53 & $92.2^{b}$ \\
\hline 11 Aug. & 35 & 46 & 98.8 & 82 & 65 & 99.3 & 67 & 56 & 95.3 \\
\hline 18 Aug. & 36 & 46 & 100.0 & 82 & 65 & 100.0 & 67 & 62 & 100.0 \\
\hline 26 Aug. & 36 & 46 & 100.0 & 82 & 65 & 100.0 & 67 & 62 & 100.0 \\
\hline
\end{tabular}

Cumulative emergence from 12 single plant emergence cages per field.

${ }^{a}$ The first visual count sampling date was 20 July (see Table 2 ).

${ }^{b}$ Date at which the late-planted strip of corn reached the Rl (silking) growth stage (Ritchie et al. 1996).

1995. After collection, western corn rootworm beetles were separated by sex. When emergence cages were placed in the fields on 19 July, several beetles were detected, but only in field 4 (the earlier-planted field).

In each field, whole-plant counts were taken as previously described to estimate beetle population densities at 4- to 7-d intervals from 28 July through 9 September 1995. Whole-plant counts were taken in the morning on each sampling date. A sampling grid was laid out across both fields that contained 45 sample sites per field. The sites ranged from 5.3 to $74.2 \mathrm{~m}$ from the dirt path that separated the fields, with more sites per unit area near the adjoining edges of each field (Fig. 1B).

Statistical Analyses. In 1994, beetle counts from each side of the center of the sampling grid were analyzed separately. In 1995, the sampling grid was laid out across both fields and each field was analyzed separately. Data in both years were collected throughout the beetle activity periods, which included different corn phenology periods and beetle population densities.

Large-scale variation was modeled using trend-surface regression analysis (Davis 1983) to describe the relationship between beetle counts and distance from the center of the late-planted strip at different corn phenology stages. Linear and quadratic trends were fit (SAS Institute 1985). Geostatistical analyses of wholeplant count data indicated that no small-scale spatial correlation was present among samples taken $\geq 5.3 \mathrm{~m}$ after 20 July 1994 (Darnell et al. 1999). This included all dates when the late-planted strip was in a phenological stage that was most attractive to beetles.

In 1995, data were analyzed using the PROC MIXED procedure (SAS Institute 1985) to quantify row, date, and row $\times$ date interactions of whole-plant counts as phenology changed in both fields. Fisher protected least significant difference (LSD) test was used to determine differences among least squares means as appropriate when a significant main effect or interaction occurred. PROC MIXED is a SAS procedure similar to the general linear model (GLM) procedure, but in the MIXED procedure effects can be fixed or random whereas all effects are treated as fixed in GLM. In each field, random sampling was executed within distances from the adjoining edges of the fields. A significance level of $P<0.05$ was used for all analyses.

\section{Results}

1994 Field Experiment. Beetle emergence profiles from early-planted corn (Table 1) indicated that by the first visual count sampling date (20 July), 50, 91, and $54 \%$ of the total beetle emergence had occurred in fields 1, 2, and 3, respectively; and gravid females were first observed in each field by $20-23$ July. When the late-planted strip reached the $\mathrm{R} 1$ (silking) growth stage (Ritchie et al. 1996), emergence was 77, 93, and $92 \%$ complete in fields 1,2 , and 3 , respectively. Field 2 had the greatest total emergence (Table 1).

In each field, no consistent distributional pattern was observed before the late-planted strip reached VT (tassel)-R1 stage (Table 2). However, after the lateplanted strip reached the VT stage (field 1) or the R1 stage (fields 2 and 3 ) and the rest of the field was $\geq \mathrm{R} 2$ stage (blister, postpollination), beetle counts became skewed toward the late-planted strip. In each case, the mean number of beetles per plant in the late-planted strip dramatically increased (Table 2). For example in field 3 , the mean number of western corn rootworms in the late-planted strip increased from 0.3 to 5.2 beetles per plant from 29 July to 5 August (Table 2). Elevated beetle counts in the late-planted strip were evident for an extended period, although this pattern was more pronounced in fields 2 and 3 than in field 1 (Table 2). Note that peak population levels occurred from 20 July to mid-August, and in late August populations began to decline in each field.

The regression equations describing the relationship between beetle counts and distance from the center of the late-planted strip became significant when the strip reached VT stage (field 1) or R1 stage (fields 2 and 3) (Table 3). The strongest relationships were seen in fields 2 and 3 (Tables 3), where the regression coefficients were not always significant on the same date on each side of the strip, but in general, similar results were observed. Highest $R^{2}$ values were 
Table 2. Mean \pm SE number of adult western corn rootworms per plant from different fields and locations in 1994

\begin{tabular}{|c|c|c|c|c|c|c|c|c|c|c|c|}
\hline \multirow{2}{*}{ Location $^{a}$} & \multirow{2}{*}{ Date } & \multicolumn{8}{|c|}{ Distance from the center of the late-planted 16 row strip $(\mathrm{m})$} & \multicolumn{2}{|c|}{ Corn phenology ${ }^{b}$} \\
\hline & & 0 & 5.3 & 10.6 & 15.9 & 21.2 & 31.8 & 53.0 & 74.2 & Field & Strip \\
\hline \multicolumn{12}{|l|}{ Field 1} \\
\hline \multirow[t]{11}{*}{ East } & 20 July & $0.7 \pm 0.2$ & $2.2 \pm 0.4$ & $1.9 \pm 0.5$ & $1.0 \pm 0.3$ & $2.0 \pm 0.4$ & $3.7 \pm 0.3$ & $0.6 \pm 0.3$ & $0.4 \pm 0.2$ & $\mathrm{Rl}$ & V13 \\
\hline & 25 July & $2.9 \pm 0.4$ & $3.8 \pm 0.5$ & $1.2 \pm 0.4$ & $2.6 \pm 0.4$ & $0.8 \pm 0.2$ & $0.8 \pm 0.3$ & $0.5 \pm 0.3$ & $0.8 \pm 0.2$ & $\mathrm{R} 2$ & VT \\
\hline & 29 July & $1.4 \pm 0.3$ & $0.8 \pm 0.2$ & $0.9 \pm 0.3$ & $1.7 \pm 0.3$ & $1.7 \pm 0.3$ & $0.8 \pm 0.4$ & $1.2 \pm 0.4$ & $0.8 \pm 0.3$ & $\mathrm{R} 2$ & $\mathrm{R} 1$ \\
\hline & 4 Aug. & $1.0 \pm 0.2$ & $0.7 \pm 0.3$ & $1.2 \pm 0.4$ & $1.1 \pm 0.4$ & $0.9 \pm 0.4$ & $1.1 \pm 0.3$ & $0.6 \pm 0.3$ & $0.7 \pm 0.2$ & R3 & $\mathrm{R} 2$ \\
\hline & 8 Aug. & $1.7 \pm 0.3$ & $0.2 \pm 0.1$ & $1.1 \pm 0.3$ & $0.4 \pm 0.2$ & $0.4 \pm 0.2$ & $0.3 \pm 0.2$ & $0.3 \pm 0.2$ & $0.2 \pm 0.1$ & R3 & $\mathrm{R} 2$ \\
\hline & 12 Aug. & $1.1 \pm 0.3$ & $0.9 \pm 0.3$ & $0.2 \pm 0.1$ & $0.5 \pm 0.2$ & $0.4 \pm 0.2$ & $0.4 \pm 0.3$ & $0.0 \pm 0.0$ & $0.1 \pm 0.1$ & R3 & $\mathrm{R} 2$ \\
\hline & 17 Aug. & $0.8 \pm 0.3$ & $0.6 \pm 0.3$ & $0.3 \pm 0.2$ & $0.5 \pm 0.3$ & $0.5 \pm 0.2$ & $0.4 \pm 0.2$ & $0.0 \pm 0.0$ & $0.3 \pm 0.2$ & $\mathrm{R} 4$ & R3 \\
\hline & 22 Aug. & $0.3 \pm 0.1$ & $0.8 \pm 0.3$ & $0.8 \pm 0.3$ & $0.1 \pm 0.1$ & $0.5 \pm 0.2$ & $0.3 \pm 0.2$ & $0.2 \pm 0.1$ & $0.1 \pm 0.1$ & R5 & $\mathrm{R} 4$ \\
\hline & 26 Aug. & $0.5 \pm 0.2$ & $0.6 \pm 0.3$ & $0.1 \pm 0.1$ & $0.3 \pm 0.2$ & $0.5 \pm 0.2$ & $0.3 \pm 0.2$ & $0.1 \pm 0.1$ & $0.3 \pm 0.2$ & R5 & R5 \\
\hline & 31 Aug. & $0.3 \pm 0.2$ & $0.0 \pm 0.0$ & $0.1 \pm 0.1$ & $0.2 \pm 0.1$ & $0.1 \pm 0.1$ & $0.1 \pm 0.1$ & $0.2 \pm 0.1$ & $0.1 \pm 0.1$ & R5 & R5 \\
\hline & 7 Sept. & $0.2 \pm 0.1$ & $0.3 \pm 0.2$ & $0.0 \pm 0.0$ & $0.2 \pm 0.1$ & $0.0 \pm 0.0$ & $0.0 \pm 0.0$ & $0.0 \pm 0.0$ & $0.1 \pm 0.1$ & $\mathrm{R} 6$ & R5 \\
\hline \multirow[t]{11}{*}{ West } & 20 July & $0.7 \pm 0.2$ & $1.9 \pm 0.4$ & $0.5 \pm 0.2$ & $0.6 \pm 0.2$ & $0.3 \pm 0.2$ & $0.3 \pm 0.2$ & $1.4 \pm 0.3$ & $1.9 \pm 0.4$ & $\mathrm{Rl}$ & V13 \\
\hline & 25 July & $2.9 \pm 0.4$ & $1.6 \pm 0.3$ & $1.2 \pm 0.4$ & $1.7 \pm 0.8$ & $0.2 \pm 0.1$ & $0.5 \pm 0.2$ & $0.2 \pm 0.1$ & $0.2 \pm 0.1$ & $\mathrm{R} 2$ & VT \\
\hline & 29 July & $1.4 \pm 0.3$ & $0.6 \pm 0.3$ & $0.5 \pm 0.2$ & $0.8 \pm 0.2$ & $0.9 \pm 0.3$ & $1.3 \pm 0.4$ & $1.3 \pm 0.4$ & $1.1 \pm 0.4$ & $\mathrm{R} 2$ & $\mathrm{R} 1$ \\
\hline & 4 Aug. & $1.0 \pm 0.2$ & $1.2 \pm 0.3$ & $0.7 \pm 0.2$ & $0.9 \pm 0.2$ & $0.9 \pm 0.4$ & $0.6 \pm 0.2$ & $0.3 \pm 0.2$ & $1.0 \pm 0.3$ & R3 & $\mathrm{R} 2$ \\
\hline & 8 Aug. & $1.7 \pm 0.3$ & $1.7 \pm 0.3$ & $0.9 \pm 0.3$ & $0.7 \pm 0.3$ & $0.6 \pm 0.2$ & $0.8 \pm 0.4$ & $0.1 \pm 0.1$ & $0.3 \pm 0.2$ & R3 & $\mathrm{R} 2$ \\
\hline & 12 Aug. & $1.1 \pm 0.3$ & $0.7 \pm 0.3$ & $0.5 \pm 0.2$ & $0.4 \pm 0.2$ & $0.8 \pm 0.2$ & $0.9 \pm 0.2$ & $0.2 \pm 0.1$ & $0.2 \pm 0.1$ & R3 & $\mathrm{R} 2$ \\
\hline & 17 Aug. & $0.8 \pm 0.3$ & $1.0 \pm 0.3$ & $0.6 \pm 0.2$ & $0.6 \pm 0.2$ & $0.7 \pm 0.2$ & $0.4 \pm 0.1$ & $0.0 \pm 0.0$ & $0.6 \pm 0.2$ & R4 & R3 \\
\hline & 22 Aug. & $0.3 \pm 0.1$ & $0.3 \pm 0.2$ & $0.2 \pm 0.1$ & $0.1 \pm 0.1$ & $0.0 \pm 0.0$ & $0.0 \pm 0.0$ & $0.1 \pm 0.1$ & $0.4 \pm 0.2$ & R5 & $\mathrm{R} 4$ \\
\hline & 26 Aug. & $0.5 \pm 0.2$ & $0.1 \pm 0.1$ & $0.0 \pm 0.0$ & $0.1 \pm 0.1$ & $0.1 \pm 0.1$ & $0.1 \pm 0.1$ & $0.2 \pm 0.2$ & $0.2 \pm 0.1$ & R5 & R5 \\
\hline & 31 Aug. & $0.3 \pm 0.2$ & $0.2 \pm 0.1$ & $0.0 \pm 0.0$ & $0.0 \pm 0.0$ & $0.0 \pm 0.0$ & $0.0 \pm 0.0$ & $0.0 \pm 0.0$ & $0.1 \pm 0.1$ & R5 & R5 \\
\hline \multirow{2}{*}{\multicolumn{12}{|c|}{ Field $2^{c}$}} \\
\hline & & & & & & & & & & & \\
\hline \multirow[t]{8}{*}{ North } & 20 July & $1.9 \pm 0.3$ & $2.1 \pm 0.4$ & $2.1 \pm 0.5$ & $1.0 \pm 0.2$ & $0.3 \pm 0.2$ & $1.6 \pm 0.3$ & $0.7 \pm 0.2$ & & $\mathrm{Rl}$ & VT \\
\hline & 25 July & $7.1 \pm 0.6$ & $3.4 \pm 0.5$ & $1.2 \pm 0.3$ & $1.0 \pm 0.3$ & $1.4 \pm 0.3$ & $1.4 \pm 0.3$ & $0.5 \pm 0.2$ & & $\mathrm{R} 2$ & $\mathrm{R} 1$ \\
\hline & 29 July & $6.2 \pm 0.8$ & $1.9 \pm 0.4$ & $2.4 \pm 0.3$ & $2.0 \pm 0.4$ & $1.2 \pm 0.4$ & $1.1 \pm 0.2$ & $0.7 \pm 0.3$ & & R3 & $\mathrm{R} 2$ \\
\hline & 4 Aug. & $3.0 \pm 0.3$ & $2.6 \pm 0.6$ & $0.4 \pm 0.2$ & $1.4 \pm 0.5$ & $1.2 \pm 0.4$ & $1.1 \pm 0.4$ & $0.8 \pm 0.3$ & & R3 & $\mathrm{R} 2$ \\
\hline & 12 Aug. & $3.9 \pm 0.6$ & $1.1 \pm 0.2$ & $0.5 \pm 0.2$ & $0.3 \pm 0.2$ & $0.2 \pm 0.1$ & $0.6 \pm 0.3$ & $0.3 \pm 0.2$ & & R3 & R3 \\
\hline & 17 Aug. & $1.4 \pm 0.3$ & $0.6 \pm 0.2$ & $0.2 \pm 0.1$ & $0.4 \pm 0.2$ & $0.2 \pm 0.1$ & $0.3 \pm 0.2$ & $0.5 \pm 0.2$ & & $\mathrm{R} 4$ & R3 \\
\hline & 22 Aug. & $0.8 \pm 0.2$ & $0.3 \pm 0.2$ & $0.1 \pm 0.1$ & $0.3 \pm 0.2$ & $0.1 \pm 0.1$ & $0.2 \pm 0.1$ & $0.1 \pm 0.1$ & & R5 & $\mathrm{R} 4$ \\
\hline & 26 Aug. & $0.5 \pm 0.2$ & $0.3 \pm 0.2$ & $0.2 \pm 0.1$ & $0.0 \pm 0.0$ & $0.0 \pm 0.0$ & $0.1 \pm 0.1$ & $0.0 \pm 0.0$ & & $\mathrm{R} 6$ & R5 \\
\hline \multirow[t]{8}{*}{ South } & 20 July & $1.9 \pm 0.3$ & $3.3 \pm 0.6$ & $3.7 \pm 0.3$ & $3.4 \pm 0.7$ & $1.4 \pm 0.4$ & $0.7 \pm 0.2$ & $1.1 \pm 0.2$ & $1.0 \pm 0.3$ & $\mathrm{R} 1$ & VT \\
\hline & 25 July & $7.1 \pm 0.6$ & $3.5 \pm 0.5$ & $3.4 \pm 0.3$ & $1.9 \pm 0.4$ & $1.3 \pm 0.4$ & $1.0 \pm 0.3$ & $1.5 \pm 0.5$ & $0.7 \pm 0.2$ & $\mathrm{R} 2$ & R1 \\
\hline & 29 July & $6.2 \pm 0.8$ & $5.0 \pm 0.8$ & $2.7 \pm 0.5$ & $2.7 \pm 0.2$ & $2.6 \pm 0.2$ & $1.0 \pm 0.3$ & $0.2 \pm 0.1$ & $0.0 \pm 0.0$ & R3 & $\mathrm{R} 2$ \\
\hline & 4 Aug. & $3.0 \pm 0.3$ & $1.1 \pm 0.3$ & $0.8 \pm 0.4$ & $0.9 \pm 0.5$ & $0.5 \pm 0.3$ & $1.1 \pm 0.2$ & $0.4 \pm 0.2$ & $0.2 \pm 0.2$ & R3 & $\mathrm{R} 2$ \\
\hline & $12 \mathrm{Aug}$. & $3.9 \pm 0.6$ & $1.6 \pm 0.6$ & $0.8 \pm 0.2$ & $0.8 \pm 0.2$ & $1.1 \pm 0.3$ & $0.8 \pm 0.2$ & $0.4 \pm 0.2$ & $0.1 \pm 0.1$ & R3 & R3 \\
\hline & 17 Aug. & $1.4 \pm 0.3$ & $0.7 \pm 0.2$ & $0.7 \pm 0.2$ & $0.7 \pm 0.2$ & $0.5 \pm 0.2$ & $0.4 \pm 0.2$ & $0.1 \pm 0.1$ & $0.2 \pm 0.1$ & $\mathrm{R} 4$ & R3 \\
\hline & 22 Aug. & $0.8 \pm 0.2$ & $1.5 \pm 0.3$ & $0.3 \pm 0.2$ & $0.5 \pm 0.2$ & $0.3 \pm 0.1$ & $0.6 \pm 0.3$ & $0.1 \pm 0.1$ & $0.2 \pm 0.2$ & R5 & $\mathrm{R} 4$ \\
\hline & 26 Aug. & $0.5 \pm 0.2$ & $0.2 \pm 0.1$ & $0.1 \pm 0.1$ & $0.1 \pm 0.1$ & $0.0 \pm 0.0$ & $0.1 \pm 0.1$ & $0.0 \pm 0.0$ & $0.0 \pm 0.0$ & $\mathrm{R} 6$ & R5 \\
\hline \multicolumn{12}{|l|}{ Field 3} \\
\hline \multirow[t]{10}{*}{ North } & 20 July & $0.4 \pm 0.1$ & $1.2 \pm 0.4$ & $0.8 \pm 0.3$ & $0.7 \pm 0.3$ & $0.6 \pm 0.3$ & $0.4 \pm 0.2$ & $0.3 \pm 0.2$ & $0.1 \pm 0.1$ & $\mathrm{Rl}$ & V11 \\
\hline & 25 July & $0.4 \pm 0.1$ & $0.2 \pm 0.1$ & $2.2 \pm 0.3$ & $2.2 \pm 0.3$ & $0.4 \pm 0.2$ & $1.2 \pm 0.3$ & $0.5 \pm 0.2$ & $0.5 \pm 0.2$ & $\mathrm{R} 2$ & V13 \\
\hline & 29 July & $0.3 \pm 0.1$ & $0.5 \pm 0.2$ & $1.5 \pm 0.4$ & $0.9 \pm 0.4$ & $0.5 \pm 0.2$ & $1.0 \pm 0.2$ & $0.8 \pm 0.3$ & $1.5 \pm 0.5$ & $\mathrm{R} 2$ & VT \\
\hline & 5 Aug. & $5.2 \pm 0.7$ & $1.6 \pm 0.5$ & $0.5 \pm 0.3$ & $0.2 \pm 0.1$ & $0.2 \pm 0.1$ & $0.6 \pm 0.3$ & $0.8 \pm 0.4$ & $0.5 \pm 0.2$ & R3 & $\mathrm{R} 1$ \\
\hline & 8 Aug. & $5.0 \pm 0.9$ & $0.6 \pm 0.2$ & $2.3 \pm 0.2$ & $1.4 \pm 0.3$ & $1.3 \pm 0.3$ & $0.3 \pm 0.2$ & $0.7 \pm 0.3$ & $0.3 \pm 0.2$ & R3 & $\mathrm{R} 1$ \\
\hline & 12 Aug. & $2.9 \pm 0.3$ & $1.2 \pm 0.3$ & $0.5 \pm 0.2$ & $1.0 \pm 0.3$ & $0.2 \pm 0.1$ & $0.4 \pm 0.2$ & $1.4 \pm 0.3$ & $1.6 \pm 0.3$ & R3 & $\mathrm{R} 2$ \\
\hline & 17 Aug. & $3.2 \pm 0.5$ & $0.4 \pm 0.2$ & $1.0 \pm 0.3$ & $0.6 \pm 0.2$ & $0.4 \pm 0.2$ & $1.2 \pm 0.3$ & $0.8 \pm 0.3$ & $1.7 \pm 0.3$ & $\mathrm{R} 4$ & R3 \\
\hline & 22 Aug. & $1.0 \pm 0.3$ & $0.2 \pm 0.1$ & $0.4 \pm 0.2$ & $0.3 \pm 0.2$ & $0.9 \pm 0.4$ & $0.7 \pm 0.2$ & $0.6 \pm 0.3$ & $0.6 \pm 0.2$ & R5 & $\mathrm{R} 4$ \\
\hline & 29 Aug. & $1.0 \pm 0.3$ & $0.5 \pm 0.3$ & $0.1 \pm 0.1$ & $0.0 \pm 0.0$ & $0.0 \pm 0.0$ & $0.1 \pm 0.1$ & $0.0 \pm 0.0$ & $0.0 \pm 0.0$ & R5 & R4 \\
\hline & 7 Sept. & $0.5 \pm 0.2$ & $0.3 \pm 0.2$ & $0.0 \pm 0.0$ & $0.1 \pm 0.1$ & $0.1 \pm 0.1$ & $0.1 \pm 0.1$ & $0.0 \pm 0.0$ & $0.0 \pm 0.0$ & R5 & $\mathrm{R} 4$ \\
\hline South & 20 July & $0.4 \pm 0.1$ & $1.1 \pm 0.4$ & $0.6 \pm 0.2$ & $1.1 \pm 0.2$ & $0.4 \pm 0.2$ & $1.7 \pm 0.4$ & $0.2 \pm 0.1$ & $0.5 \pm 0.2$ & $\mathrm{R} 1$ & V11 \\
\hline & 25 July & $0.4 \pm 0.1$ & $1.1 \pm 0.2$ & $1.1 \pm 0.4$ & $0.5 \pm 0.2$ & $0.9 \pm 0.2$ & $1.0 \pm 0.3$ & $0.0 \pm 0.0$ & $0.4 \pm 0.2$ & $\mathrm{R} 2$ & V13 \\
\hline & 29 July & $0.3 \pm 0.1$ & $0.4 \pm 0.2$ & $1.2 \pm 0.4$ & $1.2 \pm 0.3$ & $0.6 \pm 0.2$ & $0.8 \pm 0.3$ & $0.8 \pm 0.3$ & $0.5 \pm 0.2$ & $\mathrm{R} 2$ & VT \\
\hline & 5 Aug. & $5.2 \pm 0.7$ & $1.4 \pm 0.3$ & $0.8 \pm 0.3$ & $1.0 \pm 0.2$ & $1.8 \pm 0.4$ & $0.7 \pm 0.3$ & $0.7 \pm 0.2$ & $0.7 \pm 0.3$ & R3 & $\mathrm{R} 1$ \\
\hline & 8 Aug. & $5.0 \pm 0.9$ & $2.5 \pm 0.6$ & $0.8 \pm 0.2$ & $0.6 \pm 0.2$ & $2.1 \pm 0.5$ & $0.5 \pm 0.2$ & $0.4 \pm 0.2$ & $0.1 \pm 0.1$ & R3 & $\mathrm{R} 1$ \\
\hline & 12 Aug. & $2.9 \pm 0.3$ & $4.2 \pm 1.2$ & $1.3 \pm 0.3$ & $0.4 \pm 0.2$ & $1.3 \pm 0.6$ & $0.3 \pm 0.2$ & $0.1 \pm 0.1$ & $0.6 \pm 0.2$ & R3 & $\mathrm{R} 2$ \\
\hline & 17 Aug. & $3.2 \pm 0.5$ & $2.7 \pm 0.8$ & $0.6 \pm 0.2$ & $0.7 \pm 0.3$ & $0.7 \pm 0.2$ & $1.0 \pm 0.4$ & $0.3 \pm 0.2$ & $0.4 \pm 0.2$ & $\mathrm{R} 4$ & R3 \\
\hline & 22 Aug. & $1.0 \pm 0.3$ & $0.2 \pm 0.1$ & $0.0 \pm 0.0$ & $0.2 \pm 0.1$ & $0.1 \pm 0.1$ & $0.0 \pm 0.0$ & $0.1 \pm 0.1$ & $0.2 \pm 0.1$ & R5 & $\mathrm{R} 4$ \\
\hline & 29 Aug. & $1.0 \pm 0.3$ & $0.6 \pm 0.2$ & $0.0 \pm 0.0$ & $0.2 \pm 0.1$ & $0.0 \pm 0.0$ & $0.2 \pm 0.2$ & $0.0 \pm 0.0$ & $0.1 \pm 0.1$ & R5 & $\mathrm{R} 4$ \\
\hline & 7 Sept. & $0.5 \pm 0.2$ & $0.4 \pm 0.2$ & $0.0 \pm 0.0$ & $0.0 \pm 0.0$ & $0.1 \pm 0.1$ & $0.0 \pm 0.0$ & $0.0 \pm 0.0$ & $0.1 \pm 0.1$ & R5 & $\mathrm{R} 4$ \\
\hline
\end{tabular}

Sample sizes were $n=10$ plants, except for the late-planted strip, where $n=20$ plants; 0 and $5.3 \mathrm{~m}$ sampling distances are in the late-planted strip, the $5.3 \mathrm{~m}$ distance is near the boundary between the early- and late-planted corn.

${ }^{a}$ Location relative to the late-planted strip (e.g., north of the strip)

${ }^{b}$ Classification of corn growth stages (Ritchie et al. 1996) VT, tassel; R1, silking; R2, blister, post-pollination.

${ }^{c}$ The north side of the strip had one less sampling distance than the south side due to field size constraints. The last two sampling distances were 47.7 and $63.0 \mathrm{~m}$. 
Table 3. Summary of linear regression analyses of western corn rootworm whole plant counts by distance from the center of the late-planted 16 row strip for fields 1,2 , and 3 in 1994

\begin{tabular}{|c|c|c|c|c|}
\hline \multirow{2}{*}{ Location } & \multirow{2}{*}{ Date } & \multicolumn{3}{|c|}{ Distances $(m)$ from the center of the strip $(x)$} \\
\hline & & $R^{2}$ & Equation & $P$ \\
\hline \multicolumn{5}{|l|}{ Field 1} \\
\hline \multirow[t]{11}{*}{ East } & 20 July & 0.23 & $y=0.94+0.084 x-0.0013 x^{2}$ & $<0.001$ \\
\hline & 25 July $^{a}$ & 0.35 & $y=3.13-0.1 x+0.00097 x^{2}$ & 0.001 \\
\hline & 29 July & 0.02 & $y=1.21+0.0061 x-0.00015 x^{2}$ & 0.494 \\
\hline & 4 Aug. & 0.02 & $y=0.98+0.0019 x-0.000093 x^{2}$ & 0.660 \\
\hline & 8 Aug. & 0.20 & $y=1.35-0.053 x+0.00053 x^{2}$ & 0.008 \\
\hline & 12 Aug. & 0.20 & $y=1.0-0.036 x+0.00033 x^{2}$ & 0.042 \\
\hline & 17 Aug. & 0.06 & $y=0.72-0.02 x+0.00018 x^{2}$ & 0.253 \\
\hline & 22 Aug. & 0.03 & $y=0.44+0.00034 x-0.000074 x^{2}$ & 0.610 \\
\hline & 26 Aug. & 0.03 & $y=0.5-0.012 x+0.00012 x^{2}$ & 0.401 \\
\hline & 31 Aug. & 0.01 & $y=0.21-0.0048 x+0.000053 x^{2}$ & 0.578 \\
\hline & 7 Sept. & 0.05 & $y=0.23-0.011 x+0.00012 x^{2}$ & 0.121 \\
\hline \multirow{11}{*}{ West } & 20 July & 0.17 & $y=0.95-0.031 x+0.00061 x^{2}$ & 0.003 \\
\hline & 25 July $^{a}$ & 0.34 & $y=2.57-0.1 x+0.00099 x^{2}$ & 0.001 \\
\hline & 29 July & 0.01 & $y=1.0-0.0038 x+0.000096 x^{2}$ & 0.668 \\
\hline & 4 Aug. & 0.05 & $y=1.11-0.026 x+0.00031 x^{2}$ & 0.078 \\
\hline & 8 Aug. & 0.23 & $y=1.68-0.055 x+0.00049 x^{2}$ & 0.023 \\
\hline & 12 Aug. & 0.09 & $y=0.93-0.015 x+0.000067 x^{2}$ & 0.690 \\
\hline & 17 Aug. & 0.07 & $y=0.88-0.024 x+0.00025 x^{2}$ & 0.133 \\
\hline & 22 Aug. & 0.06 & $y=0.3-0.016 x+0.00024 x^{2}$ & 0.018 \\
\hline & 26 Aug. & 0.04 & $y=0.35-0.017 x+0.00021 x^{2}$ & 0.068 \\
\hline & 31 Aug. & 0.08 & $y=0.26-0.016 x+0.00019 x^{2}$ & 0.020 \\
\hline & 7 Sept. & 0.03 & $y=0.14-0.0065 x+0.000071 x^{2}$ & 0.246 \\
\hline \multicolumn{5}{|c|}{ g } \\
\hline \multirow{8}{*}{ North } & 20 July & 0.14 & $y=2.03-0.052 x+0.00054 x^{2}$ & 0.238 \\
\hline & 25 July $^{b}$ & 0.59 & $y=6.18-0.36 x+0.0049 x^{2}$ & $<0.001$ \\
\hline & $29 \mathrm{July}^{b}$ & 0.42 & $y=5.34-0.28 x+0.0037 x^{2}$ & $<0.001$ \\
\hline & 4 Aug. ${ }^{b}$ & 0.25 & $y=2.82-0.12 x+0.0016 x^{2}$ & 0.005 \\
\hline & 12 Aug. & 0.43 & $y=3.24-0.22 x+0.0032 x^{2}$ & $<0.001$ \\
\hline & 17 Aug. & 0.20 & $y=1.22-0.074 x+0.0012 x^{2}$ & 0.001 \\
\hline & 22 Aug. & 0.12 & $y=0.64-0.034 x+0.00047 x^{2}$ & 0.052 \\
\hline & 26 Aug. & 0.10 & $y=0.47-0.028 x+0.00038 x^{2}$ & 0.088 \\
\hline \multirow{8}{*}{ South } & 20 July & 0.15 & $y=2.73-0.035 x+0.00011 x^{2}$ & 0.732 \\
\hline & 25 July $^{b}$ & 0.57 & $y=6.1-0.25 x+0.0025 x^{2}$ & $<0.001$ \\
\hline & 29 July $^{b}$ & 0.55 & $y=5.9-0.21 x+0.0018 x^{2}$ & $<0.001$ \\
\hline & 4 Aug. ${ }^{b}$ & 0.32 & $y=2.36-0.09 x+0.00087 x^{2}$ & 0.001 \\
\hline & 12 Aug. & 0.36 & $y=3.11-0.13 x+0.0013 x^{2}$ & $<0.001$ \\
\hline & 17 Aug. & 0.21 & $y=1.24-0.043 x+0.00039 x^{2}$ & 0.025 \\
\hline & 22 Aug. & 0.11 & $y=0.9-0.023 x+0.00019 x^{2}$ & 0.256 \\
\hline & 26 Aug. & 0.09 & $y=0.4-0.02 x+0.0002 x^{2}$ & 0.074 \\
\hline \multicolumn{5}{|l|}{ Field 3} \\
\hline \multirow{10}{*}{ North } & 20 July & 0.06 & $y=0.66+0.0014 x-0.00013 x^{2}$ & 0.424 \\
\hline & 25 July & 0.09 & $y=0.66+0.038 x-0.00059 x^{2}$ & 0.005 \\
\hline & 29 July & 0.07 & $y=0.52+0.015 x-0.000051 x^{2}$ & 0.803 \\
\hline & 5 Aug. $b$ & 0.40 & $y=3.91-0.21 x+0.0023 x^{2}$ & $<0.001$ \\
\hline & 8 Aug. ${ }^{b}$ & 0.30 & $y=3.87-0.16 x+0.0016 x^{2}$ & 0.001 \\
\hline & 12 Aug. ${ }^{b}$ & 0.33 & $y=2.32-0.11 x+0.0014 x^{2}$ & $<0.001$ \\
\hline & 17 Aug. & 0.22 & $y=2.35-0.11 x+0.0014 x^{2}$ & $<0.001$ \\
\hline & 22 Aug. & 0.01 & $y=0.72-0.0089 x+0.00011 x^{2}$ & 0.560 \\
\hline & 29 Aug. & 0.17 & $y=0.81-0.044 x+0.00047 x^{2}$ & 0.005 \\
\hline & 7 Sept. & 0.08 & $y=0.38-0.017 x+0.00017 x^{2}$ & 0.119 \\
\hline \multirow[t]{10}{*}{ South } & 20 July & 0.05 & $y=0.56+0.025 x-0.00038 x^{2}$ & 0.034 \\
\hline & 25 July & 0.05 & $y=0.67+0.0094 x-0.00022 x^{2}$ & 0.183 \\
\hline & 29 July & 0.06 & $y=0.43+0.03 x-0.0004 x^{2}$ & 0.020 \\
\hline & 5 Aug. ${ }^{b}$ & 0.36 & $y=3.98-0.18 x+0.0019 x^{2}$ & $<0.001$ \\
\hline & 8 Aug. $b$ & 0.34 & $y=4.06-0.18 x+0.0017 x^{2}$ & $<0.001$ \\
\hline & 12 Aug. $b$ & 0.33 & $y=3.2-0.14 x+0.0014 x^{2}$ & $<0.001$ \\
\hline & 17 Aug. & 0.30 & $y=2.83-0.12 x+0.0012 x^{2}$ & 0.001 \\
\hline & 22 Aug. & 0.18 & $y=0.75-0.042 x+0.00048 x^{2}$ & 0.001 \\
\hline & 29 Aug. & 0.16 & $y=0.83-0.043 x+0.00046 x^{2}$ & 0.007 \\
\hline & 7 Sept. & 0.10 & $y=0.41-0.023 x+0.00026 x^{2}$ & 0.023 \\
\hline
\end{tabular}

${ }^{a}$ Late-planted strip was in the VT (tassel) stage.

${ }^{b}$ Late-planted strip was in the R1 (silking) or R2 (blister, post-pollination) stage.

observed during periods when beetle densities were highest and the late-planted strip was in the VT stage (field 1; Table 3) or the R1 and R2 growth stages (fields 2 and 3) (Table 3). Fig. 2 shows the patterns in the data and the fitted regression lines for the sampling dates that had the highest $R^{2}$ values for fields 1,2 , and 3 .

1995 Field Experiment. Beetle emergence occurred from the start of the experiment until 16 August 1995, 


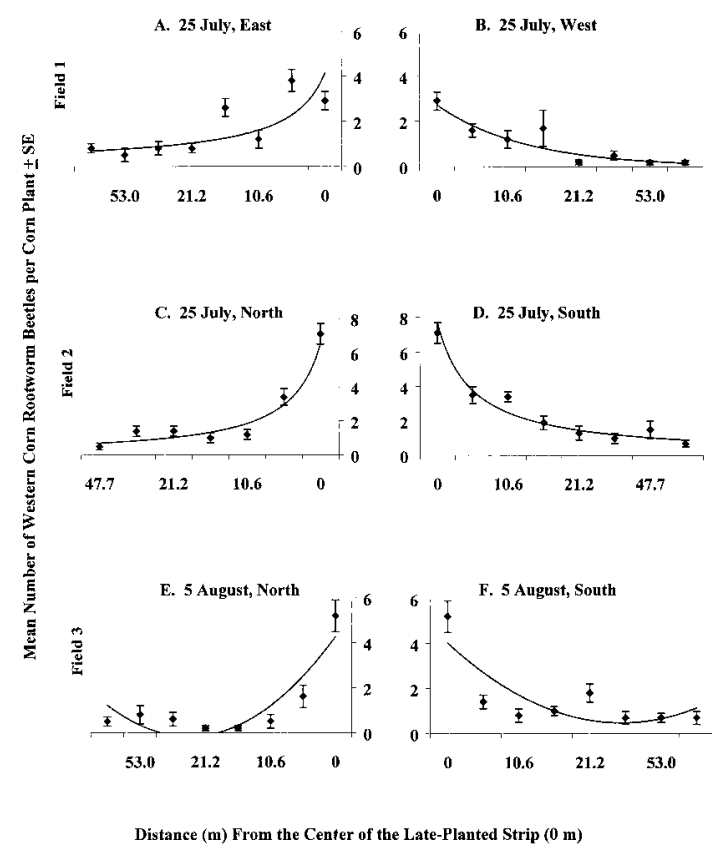

Fig. 2. Corn rootworm whole plant counts plotted against distance from the center of the late-planted strip, and the trend-surface regression lines fit to the data for field 1 (A and $B$ ), field $2(C$ and $D)$, and field $3(E$ and $F)$, for the specific sampling dates and locations in 1994. The regression equations and associated statistics are presented in Table 3.

and field 5 had the greatest total emergence (Table 4). Total emergence and emergence pattern differences between fields 4 and 5 may have been partially caused by differences in planting date. In later planted fields, as compared with earlier planted fields, initial emergence is often delayed, and total emergence is reduced (Bergman and Turpin 1984, Meinke 1995). The beetle population density was $\approx 2.5$ times greater in field 5 than in field 4 at the beginning of the experiment (Table 5).

In 1995, the MIXED procedure analyses indicated a significant date $\times$ distance interaction occurred in field $4(F=1.84 ; \mathrm{df}=35,455 ; P=0.003)$ but not in field $5(F=1.31 ; \mathrm{df}=35,455 ; P=0.117)$. There were no significant effects of row location on mean population density in fields 4 or five (field $4, F=1.19$; $\mathrm{df}=$
$7,37 ; P=0.331 ;$ field $5, F=0.89 ; \mathrm{df}=7,37 ; P=0.525)$, but densities were significantly different across dates (field $4, F=55.29 ; \mathrm{df}=5,455 ; P<0.001$; field $5, F=$ $24.27 ; \mathrm{df}=5,455 ; P<0.001)$. In field 4 , little change in beetle population levels occurred at any sampling distance from 28 July to 2 August 1995 (only two of eight sampling distances had a significant change in whole-plant counts, Table 5). However, beetle population densities increased dramatically from 2 to 9 August when field 4 reached R1 growth stage (Table $5)$. This trend was consistent across all distances sampled from the field edge adjacent to field 5 (significant increase in whole-plant counts at five of eight sampling distances, Table 5). During the transition to the R2 growth stage, 9-16 August, a significant decline in whole-plant counts occurred at many of the sampling distances (six of eight distances, Table 5). During this period, the disproportionate decrease in beetle density near the field edge (i.e., 0 - and 5.3-m distances versus other distances, Table 5) may have lead to the significant MIXED procedure distance $\times$ date interaction in field 4 . The decline in beetles per plant continued from 16 to 28 August in field 4 (Table 5: mean beetles per plant significantly declined at four of eight sampling sites) and beetle populations crashed to near zero by 6 September (Table 5).

A significant decline in mean beetles per plant occurred in field 5 after 9 August 1995 (Table 5). In contrast to field 4 , a significant increase in beetles per plant did not occur during the R1 growth stage (16-28 August) in field 5 (Table 5).

\section{Discussion}

In both years of the study, western corn rootworm beetle population dynamics were significantly impacted by corn phenological changes over time. In 1994, changes in phenology in the late-planted strip resulted in dramatic changes in beetle distribution over a short period. This trend was also evident from 2 to 9 August 1995 in field 4 during pollination. In 1994, the relative attractiveness of the late-planted strip was not limited to the $\mathrm{R} 1$ growth stage in fields 2 and 3 . Beetle populations were elevated in the strips for an extended period after pollination. This may have been because of the duration of the contrast in phenology between the strip and the surrounding field, and the beetles' preference for kernel tips and younger corn

Table 4. 1995 western corn rootworm emergence profiles

\begin{tabular}{|c|c|c|c|c|c|c|}
\hline \multirow{3}{*}{ Date } & \multicolumn{3}{|c|}{ Field 4} & \multicolumn{3}{|c|}{ Field 5} \\
\hline & \multicolumn{2}{|c|}{ Cumulative emergence } & \multirow{2}{*}{$\begin{array}{l}\text { Cumulative } \\
\% \text { emergence }\end{array}$} & \multicolumn{2}{|c|}{ Cumulative emergence } & \multirow{2}{*}{$\begin{array}{l}\text { Cumulative } \\
\% \text { emergence }\end{array}$} \\
\hline & No. $\sigma$ & No. 우 & & No. $\sigma^{*}$ & No. $q$ & \\
\hline 26 July & 2 & 1 & 6.8 & 27 & 11 & 24.5 \\
\hline 2 Aug. & 11 & 2 & 29.5 & 61 & 42 & 66.5 \\
\hline 9 Aug. & 15 & 9 & 54.5 & 83 & 51 & 86.5 \\
\hline 16 Aug. & 18 & 26 & 100.0 & 93 & 62 & 100.0 \\
\hline 28 Aug. & 18 & 26 & 100.0 & 93 & 62 & 100.0 \\
\hline
\end{tabular}

Six emergence cages per field. Emergence cages were placed in fields on 19 July 1995 . Several beetles were detected on 19 July 1995 only in the earlier planted field (field 4). 
Table 5. Mean \pm SE western corn rootworms per plant from different locations in fields 4 and 5, 1995

\begin{tabular}{|c|c|c|c|c|c|c|c|c|c|c|c|}
\hline \multirow{2}{*}{ Location } & \multirow{2}{*}{ Date } & \multicolumn{8}{|c|}{ Distance from the field edge adjacent to the dirt path that separates fields 4 and $5(\mathrm{~m})$} & \multirow{2}{*}{$\begin{array}{c}\text { Field } \\
\text { phenology }{ }^{a}\end{array}$} & \multirow{2}{*}{$\begin{array}{l}\text { Field } \\
\text { mean }^{b}\end{array}$} \\
\hline & & 0 & 5.3 & 10.6 & 15.9 & 21.2 & 31.8 & 53.0 & 74.2 & & \\
\hline \multicolumn{12}{|l|}{ Field 4} \\
\hline & 28 July & $0.8 \pm 0.3 b c$ & $1.8 \pm 0.4 \mathrm{~b}$ & $0.6 \pm 0.2 \mathrm{ab}$ & $1.2 \pm 0.4 \mathrm{bc}$ & $0.3 \pm 0.2 \mathrm{a}$ & $0.9 \pm 0.3 \mathrm{a}$ & $1.7 \pm 0.4 \mathrm{~b}$ & $2.0 \pm 0.6 \mathrm{c}$ & V14 & $1.2 \pm 0.1$ \\
\hline & 2 Aug. & $1.5 \pm 0.3 c$ & $1.6 \pm 0.4 b$ & $0.7 \pm 0.3 \mathrm{ab}$ & $1.8 \pm 0.6 \mathrm{c}$ & $1.7 \pm 0.4 \mathrm{~b}$ & $0.9 \pm 0.3 \mathrm{a}$ & $0.6 \pm 0.2 \mathrm{a}$ & $1.4 \pm 0.3 b c$ & VT & $1.3 \pm 0.1$ \\
\hline & 9 Aug. & $3.2 \pm 0.3 \mathrm{~d}$ & $2.2 \pm 0.3 b$ & $3.0 \pm 0.8 \mathrm{c}$ & $3.0 \pm 0.6 \mathrm{~d}$ & $2.3 \pm 0.4 \mathrm{~b}$ & $2.8 \pm 0.5 b$ & $3.1 \pm 0.7 \mathrm{c}$ & $2.4 \pm 0.5 \mathrm{c}$ & $\mathrm{R} 1$ & $2.8 \pm 0.2$ \\
\hline & 16 Aug. & $2.6 \pm 0.4 \mathrm{~d}$ & $0.4 \pm 0.2 \mathrm{a}$ & $1.3 \pm 0.4 b$ & $1.7 \pm 0.5 \mathrm{c}$ & $1.6 \pm 0.5 b$ & $1.1 \pm 0.3 \mathrm{a}$ & $1.7 \pm 0.3 b$ & $0.8 \pm 0.3 \mathrm{ab}$ & $\mathrm{R} 2$ & $1.4 \pm 0.2$ \\
\hline & 28 Aug. & $0.7 \pm 0.3 \mathrm{ab}$ & $0.3 \pm 0.2 \mathrm{a}$ & $0.4 \pm 0.2 \mathrm{ab}$ & $0.4 \pm 0.2 \mathrm{ab}$ & $0.2 \pm 0.1 \mathrm{a}$ & $0.6 \pm 0.2 \mathrm{a}$ & $0.6 \pm 0.3 \mathrm{a}$ & $0.1 \pm 0.1 \mathrm{a}$ & $\mathrm{R} 2$ & $0.4 \pm 0.1$ \\
\hline & 6 Sept. & $0.0 \pm 0.0 \mathrm{a}$ & $0.0 \pm 0.0 \mathrm{a}$ & $0.1 \pm 0.1 \mathrm{a}$ & $0.1 \pm 0.1 \mathrm{a}$ & $0.0 \pm 0.0 \mathrm{a}$ & $0.1 \pm 0.1 \mathrm{a}$ & $0.0 \pm 0.0 \mathrm{a}$ & $0.3 \pm 0.2 \mathrm{a}$ & R3 & $0.1 \pm 0.1$ \\
\hline \multicolumn{12}{|c|}{ 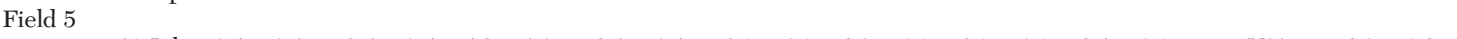 } \\
\hline & 2 Aug. & $2.8 \pm 0.4$ & $2.8 \pm 0.5$ & $0.9 \pm 0.4$ & $\begin{array}{l}3.4 \pm 1.4 \\
2.8 \pm 0.5\end{array}$ & $3.3 \pm 0.9$ & $\begin{array}{l}3.1 \pm 0.9 \\
3.3 \pm 0.6\end{array}$ & $3.0 \pm 0.5$ & $\begin{array}{l}3.4 \pm 0.0 \\
3.2 \pm 0.5\end{array}$ & V13 & $2.8 \pm 0.2 \mathrm{ab}$ \\
\hline & 9 Aug. & $1.8 \pm 0.3$ & $2.2 \pm 0.6$ & $1.7 \pm 0.6$ & $2.1 \pm 0.5$ & $3.3 \pm 0.8$ & $3.2 \pm 0.5$ & $3.1 \pm 0.8$ & $2.4 \pm 1.0$ & VT & $2.5 \pm 0.2 b$ \\
\hline & 16 Aug. & $2.0 \pm 0.3$ & $2.0 \pm 0.4$ & $1.7 \pm 0.4$ & $1.3 \pm 0.2$ & $1.2 \pm 0.3$ & $2.4 \pm 0.5$ & $2.8 \pm 0.8$ & $1.9 \pm 0.5$ & $\mathrm{R} 1$ & $1.9 \pm 0.2 \mathrm{c}$ \\
\hline & 28 Aug. & $1.6 \pm 0.4$ & $1.7 \pm 0.5$ & $1.0 \pm 0.3$ & $1.2 \pm 0.7$ & $1.0 \pm 0.3$ & $1.2 \pm 0.4$ & $1.8 \pm 0.6$ & $1.2 \pm 0.3$ & $\mathrm{R} 1$ & $1.3 \pm 0.2 \mathrm{~d}$ \\
\hline & 6 Sept. & $0.9 \pm 0.3$ & $0.7 \pm 0.4$ & $0.0 \pm 0.0$ & $0.6 \pm 0.3$ & $0.1 \pm 0.1$ & $0.6 \pm 0.3$ & $0.8 \pm 0.3$ & $0.7 \pm 0.3$ & $\mathrm{R} 2$ & $0.6 \pm 0.1 \mathrm{e}$ \\
\hline
\end{tabular}

Sample sizes were $n=10$ plants, except in the edge row that is represented by $0 \mathrm{~m}, n=20$ plants; values within columns of each subpart followed by the same letters are not significantly different $(P>0.05)$. Fisher protected LSD was performed on distance-date least squares means following a significant date $\times$ distance interaction $(F=1.85 ; \mathrm{df}=35,455 ; P=0.003)$ for field 4 . Fisher protected LSD was performed on date least squares means based on an insignificant date $\times$ distance interaction $(F=1.31 ; \mathrm{df}=35,455 ; P=0.117)$ but a significant date effect $(F=24.27 ; \mathrm{df}=5,455 ; P<0.001)$ for field 5 . Field 4 date means are included as a reference, and distance-date means are also included for field 5 as some differences are potentially biologically meaningful.

${ }^{a}$ Classification of corn growth stages (Ritchie et al. 1996) VT, tassel; R1, silking; R2, blister, post-pollination.

${ }^{b}$ Mean beetles per plant (SE) on each sampling date per field.

growth over later growth stages (Ball 1957, Hintz and George 1966). In field 4 during 1995, the rapid increase in beetle density was significant but transient, possibly because the phenological contrast between fields 4 and 5 on 9 August was not maintained.

In field 1 during 1994, population density levels in the late-planted strip never reached the levels seen in fields 2 and 3 even though field level densities were similar in fields 1-3 during most of the beetle activity period (e.g., mean beetles per plant $\pm \mathrm{SE}, 4-5$ August, field $1,0.9 \pm 0.1$; field $2,1.0 \pm 0.1$; field $3,0.8 \pm 0.1$; Table 2). Beetle densities in the strip actually declined from the VT to R1 growth stages in field 1 (Table 2) and regressions describing the relationship between beetle counts and the distance from the center of the strip were only significant on two of nine (east of strip) and three of nine (west of strip) sampling dates after the strip reached the R1 growth stage (Table 3). In field 1 , some sites within the sampling grid and several areas near the grid were weedy (e.g., foxtails, Setaria spp. and pigweeds, Amaranthus spp. were common). The foxtail and pigweed species bloomed in these areas after corn outside of the late-planted strip had pollinated. Adult western corn rootworms will readily feed on weed pollen (Ludwig and Hill 1975) and it is not uncommon to see aggregations of beetles on blooming foxtail species in and around cornfields that are past the R2 (blister) growth stage (L.J.M., unpublished data). The mosaic of alternative pollen sources in field 1 may have diluted the number of beetles found in the late-planted strip on certain sampling dates and contributed to the beetle distributional patterns recorded in field 1 , which were not as consistent over time as the patterns recorded in fields 2 and 3 .

The changes in beetle densities in both years suggest that beetles made directional movements toward pollinating corn and associated semiochemicals. In field 3 during 1994, 92\% of the emergence occurred in the early-planted corn by the time the late-planted strip reached R1 stage. Emergence was not recorded from the late-planted strip in field 3, but in another field at the same location in 1994 where emergence was compared between 3 May and 24 May planting dates, initial western corn rootworm emergence was delayed by 1 wk and total emergence was reduced 3.3-fold in the late-planted corn (Meinke 1995). Final emergence was recorded on the same date from each planting (Meinke 1995). Therefore, the late-planted strip in field 3 probably produced fewer beetles than the early-planted corn. We conclude that newly emerged beetles contributed little to the dramatic increase in beetle population levels that was observed in the late-planted strip. Furthermore, it is unlikely that a large-scale migration event occurred from outside the field because the strip was located in the middle of a relatively isolated 32 -ha field. The dramatic increase in the late-planted strip beetle counts from 0.3 beetles per plant on 29 July to 5.2 beetles per plant on 5 August appears to have occurred because of trivial movement within the field.

In 1995, directional beetle movement may be inferred when examining the interaction of fields 4 and 5 . When R1 stage was reached during the 2-9 August period in field 4 , there was a significant increase in whole-plant counts at most sites across the field (Table $5)$. The field mean increased by 1.5 beetles per plant and up to 3-5 beetles per plant at some sampling distances (Table 5). Population density levels recorded in fields 4 and 5 on 9 August (Table 5) and beetle emergence trends during the 2-9 August period (average beetles emerging per cage, field 4, 1.8; field $5,5.2$, Table 4) suggest that the population increase observed in field 4 may have been partially caused by emergence within field 4 and immigration of beetles 
from field 5 . The level of beetle emergence in field 5 may explain why the population density within field 5 did not significantly decline from 2 to 9 August.

On 16 August 1995 when field 5 began to pollinate, a significant decrease in beetle densities occurred at many sampling distances across field 4 (six out of eight distances, up to 5.5 -fold reduction; $50 \%$ reduction in the field mean, Table 5) even though an average of 3.3 beetles emerged per cage during the 9-16 August period (Table 4). A significant decline in beetle population density also occurred in field 5 ( $24 \%$ reduction in the field mean) from 9 to 16 August (Table 5) although a trend of little to no population change could be seen in field 5 at the three sampling distances closest to field 4 (0-10.6 m distances, Table 5). Beetle emergence in field 5 was similar to that recorded in field 4 during the period (field 5 : average of 3.5 beetles emerged per cage, Table 4). The lack of an apparent increase in beetle densities in field 5 during the R1 growth stage may have been the result of the seasonal period in which silking occurred. $\mathrm{R} 1$ growth stage in field 5 occurred late in the growing season (16-28 August) after emergence was over. Within-field emergence and beetle movement into field 5 may have been counteracted by the natural population decline during late August, resulting in a small net loss in beetle density. However, the contrast in phenology between field 4 and 5 , and the possible movement of beetles from field 4 into field 5 may explain why the beetle population in field 5 declined at a slower rate than the population in field 4 during late August (Table 5).

Bruss (1981) and Naranjo (1994) have suggested that random diffusion models may be adequate to describe western corn rootworm trivial movement within homogeneous cornfields. However, Naranjo (1994) documented that beetle movement is nonrandom at the boundaries of dissimilar habitats. A conceptual model that combines both random and nonrandom movement may best explain the population distribution trends that have been quantified in this study. The following hypothesis may explain how beetles were redistributed when contrasts between pollinating and nonpollinating corn were present and a trap crop effect (Hill and Mayo 1974) resulted. Random trivial movement occurs in relatively homogeneous habitat until beetles detect semiochemical plumes emitted from pollinating corn within or among fields. Directional movement takes place toward pollinating corn and pollen/silk feeding arrests beetles (e.g., 1994: field 3 in the late-planted strip). As this process continues, beetles accumulate where high quality food is concentrated. This model is consistent with our current knowledge of beetle host-finding, which is generally thought to be mediated by semiochemicals (Lampman and Metcalf 1988, Mitchell 1988, Prystupa et al. 1988, Hammack 1996). It is supported by previous work in which adult western corn rootworms preferentially oriented toward pollinating versus vegetative corn when initiating flight (Naranjo 1994) and by flight tunnel experiments where beetles made directional movements toward volatiles emitted from corn silks (Prystupa et al. 1988).

The impact of corn phenology on beetle population dynamics may affect the accuracy of decision making when an adult sampling program is used. When scouting fields, it is important to be aware of possible shortor long-term shifts in beetle densities within fields or at the interface of fields if contrasting phenologies occur side by side. If the corn growth stage in an individual field is different than surrounding cornfields, the field may act as a donor or receiver field for beetles (Meinke 1995), which may influence oviposition (Hill and Mayo 1974). Results from this study suggest that when crop phenology contrasts are present, scouting should occur at frequent intervals to reduce the possibility of missing a dramatic shift in beetle distribution. Data from 16 August 1995 in field 4 (0, 5.3-m sampling distances, Table 5) suggest that field edges should be avoided when sampling if phenological contrasts occur between adjacent cornfields. Sampling data from the field edges may not be representative of the actual field level population density. This would be especially important if an adult management strategy was being used and the timing of insecticide applications was based on whole-plant count data.

\section{Acknowledgments}

We thank J. Brown, J. Christensen, C. Darnell, B. Edins, T. Malousek, and M. Urias for field and laboratory assistance on this project. We thank R. J. Wright and B. D. Siegfried for their critical reviews of the manuscript. The research was supported in part by the University of Nebraska Agricultural Experiment Station, Project 17-058. This is paper No. 12596 of the Journal Series of the University of Nebraska Agricultural Research Division and contribution No. 1023 of the Department of Entomology, University of Nebraska.

\section{References Cited}

Abou-Fakhr, E. M., B. E. Hibbard, D. K. Jewett, and L. B. Bjostad. 1996. Electroantennogram responses of western corn rootworm (Coleoptera: Chrysomelidae) adults in relation to maize silk morphology and phenology. Environ. Entomol. 25: 430-435.

Apple, J. W., H. C. Chiang, L. M. English, L. K. French, A. J. Keaster, G. F. Krause, Z B Mayo, J. D. Munson, G. L. Musick, J. C. Owens, and others. 1977. Impact of northern and western corn rootworm larvae on field corn. North Central Regional Research Publication 239. Wisconsin Agricultural Experiment Station, Madison.

Ball, H. J. 1957. On the biology and egg-laying habits of the western corn rootworm. J. Econ. Entomol. 50: 126-128.

Bergman, M. K. and F. T. Turpin. 1984. Impact of corn planting date on the population dynamics of corn rootworms (Coleoptera: Chrysomelidae). Environ. Entomol. 13: 898-901.

Bergman, M. K., J. J. Tollefson, and P. N. Hinz. 1981. Sampling scheme for estimating populations of corn rootworm larvae. Environ. Entomol. 10: 986-990.

Bruss, R. G. 1981. Intrafield dispersal of adult corn rootworms. Ph.D. dissertation, Iowa State University. Ames, IA. 
Chiang, H. C. 1973. Bionomics of the northern and western corn rootworms. Annu. Rev. Entomol. 18: 47-72.

Coats, S. A., J. J. Tollefson, and J. A. Mutchmor. 1986. Study of migratory flight in the western corn rootworm (Coleoptera: Chrysomelidae). Environ. Entomol. 15: 1-6.

Darnell, S. J., L. J. Meinke, L. J. Young, and C. A. Gotway. 1999. Geostatistical investigation of the small-scale spatial variation of western corn rootworm (Coleoptera: Chrysomelidae) adults. Environ. Entomol. 28: 266-274.

Davis, J. C. 1983. Statistics and data analysis in geology. Wiley, New York.

Godfrey, L. D., and F. T. Turpin. 1983. Comparison of western corn rootworm (Coleoptera: Chrysomelidae) adult populations and economic thresholds in first-year and continuous corn fields. J. Econ. Entomol. 76: 1028-1032.

Godfrey, L. D., L. J. Meinke, and R. J. Wright. 1993. Vegetative and reproductive biomass accumulation in field corn: response to root injury by western corn rootworm (Coleoptera: Chrysomelidae). J. Econ. Entomol. 86: $1557-1573$.

Grant, R. H., and K. P. Seevers. 1989. Local and long-range movement of adult western corn rootworm (Coleoptera: Chrysomelidae) as evidenced by washup along southern Lake Michigan shores. Environ. Entomol. 18: 266-272.

Hammack, L. 1996. Corn volatiles as attractants for northern and western corn rootworm beetles (Coleoptera: Chrysomelidae: Diabrotica spp.). J. Chem. Ecol. 22: 12371253.

Hein, G. L., and J. J. Tollefson. 1984. Comparison of adult corn rootworm (Coleoptera: Chrysomelidae) trapping techniques as population estimators. Environ. Entomol. 13: 266-271.

Hein, G. L., and J. J. Tollefson. 1985. Use of the Pherocon AM trap as a scouting tool for predicting damage by corn rootworm (Coleoptera: Chrysomelidae) larvae. J. Econ. Entomol. 78: 200-203.

Hein, G. L., M. K. Bergman, R. G. Bruss, and J. J. Tollefson. 1985. Absolute sampling technique for corn rootworm (Coleoptera: Chrysomelidae) adult emergence that adjusts to fit common-row spacing. J. Econ. Entomol. 78: 1503-1506.

Hibbard, B. A., T. L. Randolph, E. J. Bernklau, E. M. AbouFakhr, and L. B. Bjostad. 1997. Electroantennogram-active components of maize silk for adults of the western corn rootworm (Coleoptera: Chrysomelidae). Environ. Entomol. 26: 285-296.

Hill, R. E., and Z B Mayo. 1974. Trap-crop to control corn rootworms. J. Econ. Entomol. 67: 478-450.

Hill, R. E., and Z B Mayo. 1980. Distribution and abundance of corn rootworm species as influenced by topography and crop rotation in eastern Nebraska. Environ. Entomol. 9: $122-127$.

Hintz, A. M., and B. W. George. 1966. Field cage observations of northern and western corn rootworm populations. Proc. N. Cent. Br. Entomol. Soc. Am. 21: 41.

Lampman, R. L., and R. L. Metcalf. 1988. The comparative response of Diabrotica species (Coleoptera: Chrysomelidae) to volatile attractants. Environ. Entomol. 17: 644648.

Levine, E., and H. Oloumi-Sadeghi. 1991. Management of Diabroticite rootworms in corn. Annu. Rev. Entomol. 36: $229-55$.
Ludwig, K. E., and R. E. Hill. 1975. Comparison of gut contents of adult western and northern corn rootworms in northeast Nebraska. Environ. Entomol. 4: 435-438.

Mayo, Z B, and L. L. Peters. 1978. Planting vs. cultivation time applications of granular soil insecticides to control larvae of corn rootworm in Nebraska. J. Econ. Entomol. 71: 801-803.

Meinke, L. J. 1995. Adult corn rootworm management. Univ. Neb. Agric. Res. Div. Misc. Publ. 63-C.

Mitchell, B. K. 1988. Adult leaf beetles as models for exploring the chemical basis of host-plant recognition. J. Insect Physiol. 34: 213-225.

Naranjo, S. E. 1990. Comparative flight behavior of $D i$ abrotica virgifera virgifera and Diabrotica barberi in the laboratory. Entomol. Exp. Appl. 55: 79-90.

Naranjo, S. E. 1991. Movement of corn rootworm beetles, Diabrotica spp. (Coleoptera: Chrysomelidae), at cornfield boundaries in relation to sex, reproductive status, and crop phenology. Environ. Entomol. 20: 230-240.

Naranjo, S. E. 1994. Flight orientation of Diabrotica virgifera virgifera and D. barberi (Coleoptera: Chrysomelidae) at habitat interfaces. Ann. Entomol. Soc. Am. 87: 383-394.

Pruess, K. P., J. F. Witkowski, and E. S. Raun. 1974. Population suppression of western corn rootworm by adult control with ULV malathion. J. Econ. Entomol. 67: 651655 .

Prystupa, B., C. R. Ellis, and P.E.A. Teal. 1988. Attraction of adult Diabrotica (Coleoptera: Chrysomelidae) to corn silks and analysis of the host-finding response. J. Chem. Ecol. 14: 635-651.

Ritchie, S. W., J. J. Hanway, and G. O. Benson. 1996. How a corn plant develops. Iowa Coop. Ext. Serv. Rep. 48.

Roselle, R. M. 1977. An evaluation of the Nebraska method for predicting corn rootworm (Diabrotica virgifera LeConte) larval damage based on adult sampling. M.S. thesis, University of Nebraska, Lincoln.

Ruesink, W. G. 1986. Egg sampling techniques, pp. 83-99. In J. L. Krysan and T. A. Miller [eds.], Methods for the study of pest Diabrotica. Springer, New York.

SAS Institute, 1985. SAS user's guide: statistics. SAS Institute, Cary, NC.

Spike, B. P., and J. J. Tollefson. 1991. Yield response of corn subjected to western corn rootworm (Coleoptera: Chrysomelidae) infestation and lodging. J. Econ. Entomol. 84: $1585-1590$

Stamm, D. E., Z B Mayo, J. B. Campbell, J. F. Witkowski, L. W. Andersen, and R. Kozub. 1985. Western corn rootworm (Coleoptera: Chrysomelidae) beetle counts as a means of making larval control recommendations in $\mathrm{Ne}-$ braska. J. Econ. Entomol. 78: 794-798.

Tollefson, J. J. 1990. Comparison of adult and egg sampling for predicting subsequent populations of western and northern corn rootworms (Coleoptera: Chrysomelidae). J. Econ. Entomol. 83: 574-579.

Urias, M. A. 1998. Physiological and yield responses of different types of maize to western corn rootworm larval injury. Ph.D. dissertation. University of Nebraska, Lincoln. 2000 .
Received for publication 16 April 1999; accepted 21 March 